\title{
Bronquiectasias en adultos. Características clínicas Experiencia de 5 años 1998-2003
}

\author{
JAIME CERECEDA P.*, CATALINA SAMSO Z***, \\ ALVARO SEGURA W.** y PAMELA SANHUEZA O.**
}

\author{
RETROSPECTIVE EVALUATION OF BRONCHIECTASIS \\ CHARACTERISTICS IN ADULTS AT A CHILEAN GENERAL HOSPITAL. \\ A 5 YEARS EXPERIENCE (1998-2003)
}

\begin{abstract}
In order to know the actual characteristics of bronchiectasis in an adult population, we reviewed 18 cases with this pathology confirmed by spiral CT at a general hospital (Hospital Regional de Concepción, Chile), between 1998-2003. Ten patients were males and 8 females, their mean age was $44 \pm 13.9$ years old. Most common etiologies of bronchiectasis were pulmonary tuberculosis $(44.4 \%)$ and acute pneumonia (38.8\%); main sypmtoms were chronic cough (88.8\%), persistent sputum (77.7\%) and hemoptysis (44.4\%). Spirometry detected airway obstruction in smokers $\left(F E V_{1} / F V C=58 \%\right)$ and in non smokers $\left(F E V_{1} / F V C=68 \%\right)$. Microbiological sputum exams showed Hemophilus influenzae (16.6\%), Neisseria catharralis (15\%), Pseudomona aeruginosa (13\%), Streptococcus pneumoniae (15.4\%) and Candida albicans (4\%). Spiral CT showed the following distribution of bronchiectasis: unilobular lesion 11.1\%, bilobar $72 \%$ and multilobar 16.6\%. The clinical picture of bronchiectasis in our hospital according to this up to date review, shows that our reality is essentially similar to that previously reported in literature.

Key words: Adult bronchiectasis, spiral computed tomography, etiology, clinical characteristics, microbiology.
\end{abstract}

\section{RESUMEN}

Para conocer las características actuales de las bronquiectasias en adultos en nuestro medio se revisan los 18 casos de esta patología comprobados por TAC helicoidal de tórax en el Hospital Regional de Concepción entre 1998-2003. La edad promedio de los pacientes fue de $44 \pm 13,9$ años; 10 hombres y 8 mujeres. Las etiologías más frecuentes de bronquiectasias fueron tuberculosis pulmonar $(44,4 \%)$ y neumonía aguda $(38,8 \%)$. Los síntomas más relevantes fueron tos crónica $(88,8 \%)$, expectoración persistente $(77,7 \%)$ y hemoptisis $(44,8 \%)$. La espirometría reveló alteración ventilatoria obstructiva tanto en fumadores $\left(V E F_{1} / C V F=58 \%\right)$ como en no fumadores $\left(V E F_{1} / C V F=68 \%\right)$. La bacteriología de expectoración detectó Hemophilus influenzae (16,6\%), Neisseria catharralis (15\%), Pseudomona aeruginosa (13\%), Streptococcus pneumoniae (15,4\%) y Candida albicans (4\%). La TAC de tórax demostró lesiones bilobares (72\%), multilobares $(16,6 \%)$ y unilobares $(11,1 \%)$. La realidad de las bronquiectasias en nuestro medio es que no difiere significativamente de lo descrito en la literatura.

Palabras clave: Bronquiectasias en adultos, TAC helicoidal de tórax, etiología, características clínicas, microbiología.

\footnotetext{
* Profesor Titular, Sección Enfermedades Respiratorias, Departamento de Medicina Interna, Facultad de Medicina, Universidad de Concepción.

** Alumnos Séptimo año de Medicina, Universidad de Concepción.
} 


\section{INTRODUCCIÓN}

La bronquiectasia es una enfermedad progresiva e irreversible de los bronquios mayores y bronquíolos que lleva finalmente en el tiempo a la dilatación y destrucción de áreas de la vía aérea. Esta entidad ha resurgido a raíz de la presencia de la tuberculosis y la inmunodepresión a nivel mundial ${ }^{1}$. Se produce en los pacientes una alteración de la depuración mucociliar y, por ende, acumulación de mucus en la vía aérea $^{2}$. Los bronquios se observan dilatados y obstruidos por gran cantidad de secreciones que se infectan por diversos microorganismos. La persistente inflamación e infección bronquial condiciona el llamado círculo vicioso de la enfermedad con la acumulación progresiva de mediadores inflamatorios y toxinas microbianas ${ }^{3}$. Se suceden con frecuencia exacerbaciones de estos procesos y los enfermos presentan como síntomas clásicos: tos $(90,2 \%)$, expectoración (75\%), hemoptisis $(51 \%)$, fiebre recurrente $(69,9 \%)$, neumopatia recurrente $(43,6 \%)$ y disnea $(75,5 \%)^{4}$. Las bronquiectasias se pueden clasificar en localizadas y difusas, siendo esta diferenciación útil, ya que en el caso de las localizadas que afectan sólo a un segmento o lóbulo pulmonar la terapia de elección es la quirúrgica. La causa más frecuente de bronquiectasias localizadas es la obstrucción bronquial, tales como aspiración de un cuerpo extraño, compresión extrínseca por adenopatías, estenosis de la vía aérea por anomalías estructurales de los bronquios, tumores benignos y antiguamente, por neumonía necrotizante. Las causas de bronquiectasias difusas son más numerosas, siendo sus etiologías más frecuentes las post infecciosas tales como neumonías, tuberculosis, aspergilosis broncopulmonar alérgica y las secundarias a aspiración o inhalación de substancias irritantes. Hace algunas décadas se citaba también dentro de este grupo una serie de causas post infecciosas de la infancia condicionantes de bronquiectasias, que hoy se observan con menor frecuencia, tales como coqueluche y sarampión. Más frecuente en este grupo hoy en día, son las bronquiectasias por virus influenza y adenovirus 5 . Hay bronquiectasias difusas que tienen su origen en afecciones que afectan los mecanismos de inmunidad local, tales como la fibrosis quística y la disquinesia ciliar, y bronquiectasias secundarias a fallas de mecanismos inmunológicos sistémicos, tales como la hipogamaglobulinemia y la infección por virus HIV. Existe también el clásico síndrome de Kartagener que es considerado una va- riante de la disquinesia ciliar, definiéndose por la tríada de bronquiectasias, situs inverso y sinusitis. Finalmente, existen causas misceláneas de bronquiectasias, tales como el síndrome de Young en que hay asociación con azoospermia, artritis reumatoide, síndrome de Sjögren y el "síndrome de las uñas amarillas" en que en un $40 \%$ se asocian bronquiectasias a linfáticos hipoplásicos, linfedema, derrame pleural y coloración amarilla de uñas ${ }^{4}$. Un hecho relevante es que las bronquiectasias se acompañan frecuentemente de sinusitis, por lo que el estudio respectivo debiera acompañarse también de una radiografía o una tomografia axial computada TAC de senos paranasales, si el caso lo amerita ${ }^{1}$. Las bronquiectasias se incluyen dentro de las enfermedades que condicionan limitación crónica del flujo aéreo, por lo que se debe evaluar la función ventilatoria que revela por lo general un componente obstructivo predominante, y si existe una destrucción de parénquima pulmonar, es probable un patrón mixto obstructivo y restrictivo $^{5}$. Las bronquiectasias pueden presentarse a cualquier edad, destacándose que en los países desarrollados su incidencia se ha reducido por la gran cobertura en los programas de vacunación y por la efectividad de los programas antituberculosos y disponibilidad creciente de antibióticos eficaces. No existe en la literatura cifras sobre la actual incidencia y prevalencia de la enfermedad. Los síntomas de esta afección pueden ser similares a los de la enfermedad pulmonar obstructiva crónica (EPOC), pero existen algunos hechos clínicos que las diferencian; las bronquiectasias se dan por lo general en pacientes no necesariamente fumadores, menores de 40 años y tienen mayor incidencia en mujeres en países desarrollados ${ }^{4}$. Síntomas tales como fiebre recurrente, broncorrea importante, hemoptisis y pleuresías se ven más frecuentemente en bronquiectasias que en EPOC ${ }^{1}$. En relación a epidemiología de la enfermedad, existen antecedentes de una alta prevalencia de la enfermedad en poblaciones aisladas con escaso acceso a la atención médica y alta incidencia de infecciones respiratorias durante la niñez, como se observó en un estudio en nativos de Alaska y Yukon ${ }^{5,6}$. En Chile hay sólo dos publicaciones sobre bronquiectasias en adultos en los últimos 12 años, no existiendo en estos estudios cifras sobre la verdadera prevalencia de la enfermedad ${ }^{7,8}$.

El objetivo primario de este estudio fue conocer cuán adecuadamente se diagnostican las bronquiectasias en Chile y más específicamente en la Octava Región del país. Se realizó para este objeto un estudio retrospectivo a nivel del 
hospital Regional de Concepción. Los objetivos secundarios de este estudio fueron conocer los agentes bacterianos más frecuentes, el compromiso radiológico pulmonar y los resultados del tratamiento quirúrgico.

\section{MATERIAL Y MÉTODO}

Se realizó una revisión retrospectiva de fichas clínicas del hospital Regional de Concepción con diagnóstico de bronquiectasias en pacientes adultos entre el $1^{\circ}$ de Enero de 1998 y el 30 de abril de 2003. Se recopiló un total de 195 casos que estaban en control regular en el Servicio de Respiratorio y de éstos se seleccionaron 18 que tenían comprobación de bronquiectasias mediante tomografia axial computada helicoidal (Phillips CT Secura MCT 4781, MCT 4791, MCT 4801). Se analizó en cada uno de los pacientes sus antecedentes de evolución clínica, desde la fecha del diagnóstico, antecedentes demográficos, tabaquismo, radiografia de tórax, espirometría y estudio bacteriológico del esputo, que comprendía búsqueda de aerobios, Koch y hongos. Igualmente se efectuó un análisis de los 177 pacientes cuyo diagnóstico presuntivo de bronquiectasia se hizo sólo por clínica y radiografía de tórax. No todos los pacientes estudiados tenían TAC de tórax, debido a que en nuestro hospital hubo problemas técnicos reiterados con el antiguo tomógrafo y sólo desde el año 2000 se dispuso de un moderno tomógrafo helicoidal. El estudio de función respiratoria se realizó mediante un espirómetro Collins DSII Plus, utilizando los valores teóricos de Gutiérrez y colaboradores 9 .

\section{RESULTADOS}

En la Tabla 1 se analizan las características demográficas de los 18 pacientes estudiados destacando que el promedio de edad fue de $44 \pm 14$ DS años con predominio del sexo masculino, 10 hombres y 8 mujeres. Un $50 \%$ de los pacientes eran fumadores con un promedio de $6,36 \pm 4,5$ paquetes año $(8,6 \pm 5,1$ en hombres y $3,5 \pm 1,19$ en mujeres). El tiempo promedio de evolución de la enfermedad desde el inicio de los síntomas fue de $22 \pm 9$ años. En Tabla 2 se consigna la etiología de las bronquiectasias, existiendo en el grupo estudiado un franco predominio de tuberculosis pulmonar en 8 pacientes con un $44,4 \%$ seguido por antecedentes de neumonías en 7 con un $38,8 \%$ y sólo 2 tenían antece- dentes de coqueluche en su juventud $(11,1 \%)$. Llama la atención que un paciente no tenía una causa identificable de bronquiectasia $(5,5 \%)$. En la Tabla 3 se constata que los síntomas más frecuentes fueron tos en16 pacientes $(88,8 \%)$ seguida de expectoración en $14(77 \%)$ y menos frecuentes fueron la hemoptisis en $8(44,4 \%) \mathrm{y}$

Tabla 1. Características demográficas y hábito tabáquico en pacientes con bronquiectasias

\begin{tabular}{|c|c|c|}
\hline Pacientes (n) & \multicolumn{2}{|c|}{18} \\
\hline Edad (años) & \multicolumn{2}{|c|}{$44 \pm 14$} \\
\hline \multicolumn{3}{|l|}{ Sexo $\%$} \\
\hline Masculino & \multicolumn{2}{|c|}{55,5} \\
\hline Femenino & \multicolumn{2}{|c|}{44,5} \\
\hline Tabaco (paq /años) & \multicolumn{2}{|c|}{$6,36 \pm 4,5$} \\
\hline Masculino & \multicolumn{2}{|c|}{$8,6 \pm 5,1$} \\
\hline Femenino & \multicolumn{2}{|c|}{$3,5 \pm 1,19$} \\
\hline T.E.E. (años) \# & \multicolumn{2}{|c|}{$22 \pm 9$} \\
\hline \multicolumn{3}{|c|}{$\begin{array}{l}\text { paq }=\text { paquete } \\
\text { \# tiempo de evolución de la enfermedad } \\
\text { Cada valor representa el promedio } \pm \text { su DS }\end{array}$} \\
\hline \multicolumn{3}{|c|}{$\begin{array}{l}\text { Tabla 2. Etiología de bronquiectasias } \\
\text { en } 18 \text { pacientes }\end{array}$} \\
\hline Causas & $\mathbf{n}$ & $\%$ \\
\hline TBC pulmonar & 8 & 44,4 \\
\hline Neumonías & 7 & 38,8 \\
\hline Coqueluche & 2 & 11,1 \\
\hline Desconocida & 1 & 5,5 \\
\hline Totales & 18 & 99,8 \\
\hline
\end{tabular}

Tabla 3. Síntomas y signos relevantes en 18 pacientes con bronquiectasias

\begin{tabular}{lrc}
\hline Síntomas & $\mathbf{n}$ & $\boldsymbol{\%}$ \\
\hline Tos & 16 & 88,8 \\
Expectoración & 14 & 77,7 \\
Hemoptisis & 8 & 44,4 \\
Disnea & 5 & 27,7 \\
Signos físicos & & \\
Crépitos & 14 & 77,7 \\
Sibilancias & 15 & 83,3 \\
\hline
\end{tabular}


Tabla 4. Valores espirométricos en 18 pacientes con bronquiectasias

\begin{tabular}{|c|c|c|c|c|}
\hline \multirow[b]{2}{*}{ Sexo } & \multicolumn{2}{|c|}{$\begin{array}{l}\text { Fumadores } \\
\qquad(\mathbf{n}=9)\end{array}$} & \multicolumn{2}{|c|}{$\begin{array}{l}\text { No Fumadores } \\
\quad(n=9)\end{array}$} \\
\hline & $M=6$ & $F=3$ & $M=7$ & $\mathrm{~F}=2$ \\
\hline & SBD & $\mathrm{CBD}$ & SBD & CBD \\
\hline $\mathrm{CVF}$ & $65 \pm 17$ & $66 \pm 20$ & $70 \pm 15$ & $72 \pm 14$ \\
\hline $\mathrm{VEF}_{1} \quad(\%)$ & $56 \pm 18$ & $55 \pm 14$ & $64 \pm 26$ & $66 \pm 20$ \\
\hline $\mathrm{VEF}_{1} / \mathrm{CVF}(\%)$ & $58 \pm 15$ & $60 \pm 20$ & $68 \pm 20$ & $70 \pm 14$ \\
\hline
\end{tabular}

$\mathrm{SBD}=$ sin broncodilatador $\mathrm{CBD}=$ con broncodilatador

Valores expresados en \% promedio de su valor teórico de referencia \pm su DS

p: ns al comparar valores espirométricos entre fumadores y no fumadores.

disnea en $5(27,7 \%)$. En relación a los signos clínicos al examen de tórax predominan las sibilancias en 15 enfermos $(83,3 \%)$ y crepitaciones en $14(77,7 \%)$. En relación a la función respiratoria (Tabla 4), la espirometría muestra que el $\mathrm{VEF}_{1}$ está disminuido en fumadores y no fumadores, siendo esta alteración de mayor significación en fumadores. Igualmente la relación $\mathrm{VEF}_{1} / \mathrm{CVF}$ está disminuida tanto en hombres como mujeres, siendo mayor esta alteración en hombres fumadores. Ninguno de los parámetros señalados tuvo significación estadística. La radiografía de tórax posteroanterior y lateral (Tabla 5), reveló imágenes de fibrosis retráctil con disminución del volumen pulmonar en 14 pacientes $(78 \%)$, inflamación peribronquial en 2 $(10 \%)$ e imágenes quísticas también en 2 (10\%). En relación a la extensión de las bronquiectasias la TAC de tórax (Tabla 6), mostró que estas fueron bilobares en 13 (72,2\%), multilobares en $3(17 \%)$ y unilobares en 2 $(11,1 \%)$. El sitio anatómico más frecuente de ubicación de lesiones fue el lóbulo inferior izquierdo en 7 enfermos (38,8\%) seguido por el lóbulo superior derecho con $6(33,3 \%)$ y el ló-

Tabla 5. Imagenología en 18 pacientes con bronquiectasias

\begin{tabular}{lrr}
\hline Radiografía de tórax & n & \% \\
\hline Fibrosis retráctil & 14 & 78 \\
Inflamación peribronquial & 2 & 11 \\
Imagen quística & 2 & 11 \\
Totales & 18 & 100 \\
\hline
\end{tabular}

bulo superior izquierdo con 5 (27,9\%). En relación a la bacteriología del esputo (Tabla 7), analizado mediante cultivo de expectoración efectuado a cada paciente durante un control de policlínico se confirma el Hemophilus influenzae en 3 pacientes $(16,6 \%)$, Neisseria catarralis en

Tabla 6. Tomografia axial computada de tórax en 18 pacientes con Bronquiectasias

\begin{tabular}{lrr}
\hline Extensión & n & \% \\
\hline Bilobares & 13 & 72 \\
Multilobares & 3 & 17 \\
Unilobares & 2 & 11 \\
Totales & 18 & 100 \\
Localización anatómica & & \\
$\quad$ Lóbulo inferior izquierdo & 7 & 39 \\
$\quad$ Lóbulo superior derecho & 6 & 33 \\
$\quad$ Lóbulo superior izquierdo & 5 & 28 \\
Totales & 18 & 100 \\
\hline
\end{tabular}

Tabla 7. Bacteriología del esputo en 18 pacientes con bronquiectasias

\begin{tabular}{lrr}
\hline Germen & n & \% \\
\hline Hemophilus influenzae & 3 & 16,6 \\
Neisseria catarralis & 3 & 16,6 \\
Pseudomonas aeruginosa & 2 & 11,2 \\
Streptococcus pneumoniae & 3 & 16,6 \\
Candida albicans & 2 & 11,2 \\
No consignado & 5 & 27,8 \\
Totales & 18 & 100 \\
\hline
\end{tabular}


$3(16,6 \%)$, Pseudomona aeruginosa en 2 $(11,2 \%)$, Streptococcus neumoniae en $3(16,6 \%)$ y Candida albicans en $2(11,2 \%)$. No se efectuó el estudio bacteriológico en 5 pacientes $(27,7 \%)$. Finalmente de los 18 pacientes estudiados, 7 fueron sometidos a tratamiento quirúrgico; de ellos 4 eran fumadores, su edad al momento de revisión de antecedentes fue de $33,7 \pm 9$ años y fueron operados a los $23 \pm 4$ años. En cuanto al procedimiento quirúrgico efectuado, uno fue sometido a una lobectomía inferior derecha, 5 a lobectomía inferior izquierda y uno a lobectomía media derecha. Los controles espirométricos promedio, un año después de la intervención fueron: $\mathrm{CVF}=80 \% \pm 4,7, \mathrm{VEF}_{1}=$ $74 \% \pm 2 \mathrm{y} \mathrm{VEF}_{1} / \mathrm{CVF}=73 \% \pm 3$. El tratamiento médico se efectuó fundamentalmente a aquellos pacientes que presentaban síntomas y signos de infección bronquial durante las exacerbaciones y que también se acompañaban de obstrucción bronquial. Se indicó empíricamente hidratación y antibióticos orales en base a cloranfenicol o amoxicilina-clavulánico por 14 días. En caso de no haber respuesta se efectuó estudio de expectoración con Gram y cultivo. De aislarse Pseudomonas, el paciente se hospitalizó y se inició terapia en base a ceftazidima y ciprofloxacino por 21 días. La obstrucción bronquial se trató con inhaladores $\beta$ adrenérgicos y teofilina de acción prolongada, por igual lapso de tiempo.

En relación a los 177 pacientes con diagnóstico presuntivo de bronquiectasia (Tabla 8), estos tuvieron un promedio de edad de $50 \pm 12$ años con predominio del sexo masculino $56,5 \%$.

Tabla 8. Características demográficas y hábito tabáquico en pacientes con bronquiectasias sin TAC de tórax

\begin{tabular}{ll}
\hline Pacientes (n) & 177 \\
Edad (años) & $50 \pm 12$ \\
Sexo \% & \\
Masculino & 56,5 \\
Femenino & 43,5 \\
Tabaco (paq/años) & $7,4 \pm 15$ \\
Masculino & $9,4 \pm 4,1$ \\
Femenino & $3,8 \pm 6.5$ \\
TEE* (años) & $24 \pm 7$ \\
\hline
\end{tabular}

paq $=$ paquete

*tiempo de evolución de la enfermedad

- Cada valor representa el promedio \pm su DS
El hábito tabáquico expresado en paquetes/año señaló un promedio de 7,4 \pm 15 correspondiendo $9,4 \pm 4,1$ a hombres y 3,8 $\pm 6,5$ a mujeres. El tiempo de evolución de la enfermedad fue de $24 \pm 7$ años. En cuanto a etiología de las bronquiectasias (Tabla 9), se evidenció predominio de tuberculosis pulmonar, confirmada por baciloscopias positivas en el esputo en 88 pacientes (55\%), neumonías en 56 (32\%) y coqueluche en $33(4,5 \%)$. Los síntomas y signos clínicos principales (Tabla 10), fueron expectoración en 150 pacientes $(84,7 \%)$ y tos en $130(73,4 \%)$. La signología pulmonar estuvo representada por crepitaciones en 159 enfermos $(90 \%)$ y sibilancias en $18(10 \%)$. En relación a la radiografía de tórax, anteroposterior y lateral (Tabla 11), se observó en los 177 pacientes inflamación peribronquial en 94 pacientes (53\%) e imágenes quísticas en 83 (47\%). La bacteriología de expectoración se pudo efectuar a sólo 140 enfermos y permitió aislar Hemophilus influenzae en 70 pacientes $(39,5 \%)$ seguido por Neisseria catharralis en $39(17,4 \%)$ y Streptococcus pneumoniae en $31(22,1 \%)$. No se detecto germen en 37 pacientes $(21 \%)$. La función respiratoria en estos pacientes (Tabla 12), indica un patrón obstructivo tanto en fumadores como en no fumadores siendo más acentuado

Tabla 9. Etiología de bronquiectasias en 177 pacientes sin TAC de tórax

\begin{tabular}{lrr}
\hline Causa & n & \% \\
\hline TBC pulmonar & 88 & 55 \\
Neumonía & 56 & 32 \\
Coqueluche & 33 & 13 \\
Totales & 177 & 100 \\
\hline
\end{tabular}

Tabla 10. Síntomas y signos respiratorios en 177 pacientes con bronquiectasias sin TAC de tórax

\begin{tabular}{lrr}
\hline & $\mathbf{n}$ & $\%$ \\
\hline Síntomas & & \\
$\quad$ Expectoración & 150 & 84,7 \\
Tos & 130 & 73,7 \\
& & \\
Signos & & \\
Crepitaciones & 159 & 90 \\
Sibilancias & 18 & 10 \\
\hline
\end{tabular}


Tabla 11. Imagenología y bacteriología del esputo en 177 pacientes sin TAC de tórax

\begin{tabular}{lrc}
\hline & n & $\%$ \\
\hline Radiografía de tórax & & \\
Inflamación peribronquial & 94 & 53 \\
Imágenes quísticas & 83 & 47 \\
Totales & 177 & 100 \\
Bacteriología del esputo & & \\
Hemophilus influenzae & 70 & 39,5 \\
Neisseria catarralis & 39 & 22 \\
Streptococcus pneumoniae & 31 & 17,5 \\
Sin bacteriología & 37 & 21 \\
Totales & 177 & 100 \\
\hline
\end{tabular}

en fumadores $\left(\mathrm{VEF}_{1}=46 \% \pm 24\right)$. No hubo diferencias significativas entre pacientes fumadores y no fumadores al evaluar CVF, $\mathrm{VEF}_{1} \mathrm{y}$ $\mathrm{VEF}_{1} / \mathrm{CVF}$. De los 177 pacientes se practicó cirugía en 25 casos (14\%) correspondiendo a pacientes con bronquiectasias secundarias a tuberculosis pulmonar en $10(40 \%)$ y secundarias a neumonías en 15 (60\%) (Tabla 13). La indicación quirúrgica estuvo dada fundamentalmente por hemoptisis recidivante, efectuándose bilobectomias en 15 pacientes y unilobectomia en 10. En 2003 sólo están en control en el policlínico del Hospital Regional Concepción, 15 de estos pacientes intervenidos, siendo su evolución satisfactoria. El resto de ellos se controla en hospitales periféricos de la VIII Región.

Tabla 12. Valores espirométricos en 177 pacientes con bronquiectasias sin TAC de tórax

\begin{tabular}{|c|c|c|c|c|c|}
\hline \multirow[b]{2}{*}{ Sexo } & \multicolumn{2}{|c|}{$\begin{array}{c}\text { Fumadores } \\
\mathrm{n}=\mathbf{1 0 0}\end{array}$} & \multicolumn{2}{|c|}{$\begin{array}{l}\text { No fumadores } \\
n=77\end{array}$} & \multirow{2}{*}{$\begin{array}{c}\text { p fumadores } \\
\text { versus } \\
\text { no fumadores }\end{array}$} \\
\hline & $M=60$ & $F=40$ & $M=40$ & $\mathbf{F}=37$ & \\
\hline & SBD & CBD & SBD & CBD & \\
\hline $\operatorname{CVF}(\%)$ & $63 \pm 20$ & $62 \pm 18$ & $69 \pm 20$ & $70 \pm 16$ & $\mathrm{~ns}$ \\
\hline $\mathrm{VEF}_{1}(\%)$ & $46 \pm 24$ & $50 \pm 12$ & $53 \pm 14$ & $55 \pm 18$ & $\mathrm{~ns}$ \\
\hline $\mathrm{VEF}_{1} / \mathrm{CVF}(\%)$ & $59 \pm 19$ & $60 \pm 15$ & $66 \pm 18$ & $67 \pm 13$ & $\mathrm{~ns}$ \\
\hline
\end{tabular}

$\mathrm{SBD}=\sin$ broncodilatador, $\mathrm{CBD}=$ con broncodilatador

Valores expresados en $\%$ de su valor teórico de referencia \pm su DS

p: ns al comparar valores espirométricos entre fumadores y no fumadores.

Tabla 13. Cirugía en 25 pacientes con bronquiectasias secundarias sin TAC de tórax

\begin{tabular}{lrr}
\hline Causa & n & \% \\
\hline TBC pulmonar & 10 & 40 \\
Neumonías & 15 & 60 \\
Totales & 25 & 100 \\
\hline
\end{tabular}

\section{DISCUSIÓN}

Esta experiencia clínica estudió 18 casos con diagnóstico de certeza de bronquiectasias. En este estudio hubo predominio del sexo masculino (55\%) siendo fumadores el $50 \%$ de los pacientes estudiados. La edad promedio del grupo estudiado fue de $44 \pm 13,9$ siendo esta edad más baja que otra serie similar que fue de
$57,2 \pm 16,7^{12}$. No tenemos explicación clara a este hecho ya que no constatamos en los casos estudiados antecedentes de deficiencias inmunológicas, ni de fibrosis quística que se observan a edad temprana ${ }^{1}$. El tiempo promedio de evolución de la enfermedad fue de $22 \pm 9$ años que es muy similar a otra serie que indica que transcurren aproximadamente 20 años después del episodio inicial y la aparición de síntomas ${ }^{13}$. Al igual que otras experiencias, la principal sintomatología de nuestros pacientes fué en orden decreciente de acuerdo a incidencia: tos, expectoración y hemoptisis, no observándose episodios de pleuresías recurrentes como se comunica en otras series 5 .

En relación a la etiología de las bronquiectasias, la gran mayoría de nuestros pacientes tenían antecedentes de infecciones respiratorias previas, ocupando la tuberculosis pulmonar el primer lugar, lo que no es extraño en Concepción, dado que la incidencia de la tuberculosis pulmonar 
con baciloscopias positivas ha oscilado entre 74,8 y 15,1 por 100.000 habitantes en las últimas 3 décadas ${ }^{14}$. En un estudio efectuado en Estados Unidos, la tuberculosis junto con la sarcoidosis ocupan el tercer lugar como patologías predisponentes de bronquiectasias ${ }^{12}$. Llama la atención en nuestra experiencia clínica que en un $11,1 \%$ no se pesquisó etiología, cifra bastante inferior a otras experiencias similares en que no se encontró la causa de las bronquiectasias en un 53 $\mathrm{y}$ en un $30,1 \%$ de los casos $(15,6)$. La posible explicación a lo anterior es que nuestra muestra estudiada fue reducida. En relación a la función respiratoria evaluada mediante espirometría, hubo franco predominio de limitación funcional obstructiva en un 55\% tanto en pacientes fumadores como no fumadores, debiéndose probablemente esta alteración a causas ya citadas por otros autores, tales como, daño anatómico de la vía aérea periférica, presencia de secreciones bronquiales y posiblemente en algunos casos, a hiperreactividad bronquial ${ }^{15}$. En un $22 \%$ de los casos se encontró limitación restrictiva, que correspondió a aquellos pacientes con mayor daño anatómico del parénquima pulmonar, hecho que también es mencionado en otras experiencias 5 . Los pacientes con espirometría normal correspondieron a 5 pacientes $(22 \%)$, con un promedio de edad de $34,4 \pm 8$ no fumadores con antecedentes de neumonía en su juventud comprobados por radiografía de tórax. Se hace mención de la excelente evolución clínica de los pacientes operados con bronquiectasias localizadas a una edad temprana en el curso de la enfermedad, traducida por los valores espirométricos postoperatorios. Lo anterior está de acuerdo con la literatura, que indica que en las condiciones señaladas, el tratamiento quirúrgico de las bronquiectasias, permite una remisión de síntomas en un $90 \%$ de los casos en un seguimiento a 4 y 6 años, con una mortalidad perioperatoria $<3 \%$. En relación al estudio imagenológico de tórax, si bien es cierto las radiografías fueron todas sugerentes de bronquiectasias, el diagnóstico de certeza se obtuvo mediante TAC de tórax. Dado que el promedio de edad de nuestros pacientes fue de $44 \pm 13$ años, no hubo prácticamente dudas del diagnóstico diferencial radiológico entre bronquiectasias con EPOC como citan algunos autores ${ }^{12}$. En relación a la bacteriología de expectoración, las cepas aisladas de nuestros enfermos correspondieron a las que habitualmente colonizan el tracto respiratorio de pacientes con bronquiectasias, tales como Hemophilus influenzae, Streptococus pneumoniae y Neisserias catarralis. Cabe desta- car que en menor proporción se aisló Pseudomonas Aeruginosa y Candida, que no correspondieron en nuestra casuística a pacientes con bronquiectasias secundarias a fibrosis quística como ha sido señalado en la literatura ${ }^{16}$. Finalmente, al comparar los pacientes con certeza diagnóstica de bronquiectasia mediante TAC helicoidal con aquellos sin TAC podemos decir que ambos grupos se comportan de manera muy similar en cuanto a las características etarias, tabaquismo, bacteriologia y función respiratoria.

\section{CONCLUSIONES}

Reconocemos que el presente estudio presenta una casuística muy reducida como que también carece de un estudio inmunológico inicial, pese a que clínicamente no hubo motivos para sospechar de esta causal en los enfermos estudiados. Sin embargo, nos parece que la realidad clínico-radiológica, bacteriológica y funcional respiratoria de los pacientes con bronquiectasias en nuestro hospital, no difiere significativamente de lo descrito en la literatura. Se cumplió con el objetivo inicial de presentar las características de esta patología en el hospital Regional de Concepción y al mismo tiempo se actualizan conceptos sobre bronquiectasias, una enfermedad que se confunde comúnmente con el asma bronquial y la EPOC siendo por ende subdiagnosticada.

\section{BIBLIOGRAFÍA}

1.- STANLEY B F. Bronchiectasis The changing clinical scenario. J Respir Dís 2000; 21: 666-81.

2.- BARKER A F, BARDANA E J. Bronchiectasis: Update of an orphan disease. Am J Respir Dis 1988; 137: 969 78.

3.- ANGRILL J, AGUSTI C, DE CELIS R, FILELLA X, RAÑO A, ELENA $M$ et al. Bronquial inflamation in patients with clinically stable bronchiectasis. Am J Respir Crit Care Med 2001; 164: 1628-16.

4.- DELEN F M, BARKER A F. New concepts in diagnosis and management of bronchiectasis. Semin Respir Crit Care Med 1999; 20: 311-20.

5.- BARKER A F. Bronchiectasis. N Engl J Med 2002; 346: 1383-93.

6.- SINGLETON R, MARRIS A, REDDING G. Bronchiectasis in Alaska native children; causes in clinical courses. Pediatric Pulmonology 2000; 29: 182 7.

7.- COSTA M, WHITTLE S. Bronquiectasias. Boletín Hospital S J de Dios 1993; 40: 24-9.

8.- CORRALES R, PIDDO C, RIBERO S, TALESNIK E. Bronquiectasia como forma de presentación de inmunodeficiencía común variable. Rev Chil Enf Respir 1992; 8: 63-8. 
9.- GUTIÉRREZ M, RIOSECO F, ROJAS A, CASANOVA D. Determinación de valores espirométricos en una población normal mayor de 5 años a nivel del mar. Rev Méd Chile 1996: 124; 1295-306.

10.- PERRY K M A, KING D S. Bronchiectasis: a study of prognosis based on a follow up of 44 patients. Am Rev Tuberc 1940; 41: 531-48

11.- KONIETZKO N F J, CARTON R W, LEROY E P. Causes of death in patients with bronchiectasis. Am Rev Respir Dis 1969; 100: 852-8.

12.- BROOKE N, RIBERA M, DALE A, SHEPERD R, CARTER R. Clinical, pathophysiologic and microbiologie characterisation of bronchiectasis in an aging cohort. Chest 1995; 108: 955-61.

13.- MYSLINIEC V, PINA J S. Brochiectasis the other obstructive lung disease. Postgrad Med 1999; 106: 12331.

14.- Departamento Epidemiología. Ministerio de Salud, VIII Región.

15.- PASTEUR M, HELLIWEIL S, HOUGLITON S, WEBB J, FOWERAKER J,COULDEN R, et al. An investigation into causative factors in patients with bronchiectasis. Am J Respir Crit Care Med 2000; 162: 1277-48.

16.- PARÉ P D. Synopsis of diseases of the chest. W B. Saunders Company 1994; 677-87. 